Supporting Information Available

\title{
Evaluation of Computational Chemistry Methods: Crystallographic and Cheminformatics Analysis of Aminothiazole
} Methoximes

Tulay Ercanli and Donald B. Boyd*

Department of Chemistry, Indiana University-Purdue University at Indianapolis, 402 North Blackford Street, Indianapolis, IN 462023274

* To whom correspondence should be addressed. E-mail: boyd@chem.iupui.edu. 
Table S1. Experimental Bond Lengths (in angstroms) of ATMO-containing Structures in the Cambridge Structural Database

\begin{tabular}{lccccccccccc} 
CSD code & N2-C1 & C1-N1 & N1-C2 & C2-C3 & C3-S1 & S1-C1 & C2-C4 & C4-N3 & N3-O1 & C4-C5 & C5-O2 \\
\hline BAGSAD & 1.301 & 1.348 & 1.388 & 1.337 & 1.735 & 1.718 & 1.476 & 1.290 & 1.377 & 1.522 & 1.212 \\
BAGSEH1 & 1.317 & 1.322 & 1.388 & 1.351 & 1.726 & 1.735 & 1.447 & 1.291 & 1.395 & 1.524 & 1.244 \\
BAGSEH2 & 1.319 & 1.342 & 1.406 & 1.334 & 1.727 & 1.728 & 1.459 & 1.270 & 1.402 & 1.531 & 1.233 \\
CFTXIM & 1.335 & 1.304 & 1.388 & 1.353 & 1.714 & 1.755 & 1.457 & 1.280 & 1.406 & 1.511 & 1.195 \\
EAMTHZ & 1.322 & 1.341 & 1.388 & 1.345 & 1.722 & 1.707 & 1.466 & 1.285 & 1.387 & 1.521 & 1.193 \\
EATZAC & 1.343 & 1.308 & 1.391 & 1.345 & 1.715 & 1.737 & 1.458 & 1.288 & 1.382 & 1.505 & 1.187 \\
FAJMAE & 1.341 & 1.329 & 1.399 & 1.332 & 1.728 & 1.727 & 1.487 & 1.288 & 1.406 & 1.465 & 1.241 \\
FAJMEI & 1.331 & 1.326 & 1.407 & 1.331 & 1.731 & 1.723 & 1.467 & 1.290 & 1.402 & 1.501 & 1.215 \\
FUPKOQ & 1.345 & 1.304 & 1.385 & 1.341 & 1.716 & 1.751 & 1.454 & 1.282 & 1.401 & 1.518 & 1.190 \\
GAFTIQ & 1.341 & 1.304 & 1.385 & 1.364 & 1.733 & 1.756 & 1.453 & 1.294 & 1.399 & 1.513 & 1.234 \\
GOHRAW & 1.336 & 1.316 & 1.422 & 1.334 & 1.729 & 1.724 & 1.462 & 1.273 & 1.397 & 1.516 & 1.226 \\
VENWIU & 1.281 & 1.342 & 1.396 & 1.311 & 1.744 & 1.725 & 1.442 & 1.278 & 1.428 & 1.566 & 1.228 \\
Mean & 1.326 & 1.324 & 1.395 & 1.340 & 1.727 & 1.732 & 1.461 & 1.284 & 1.399 & 1.516 & 1.217 \\
Std Dev & 0.019 & 0.017 & 0.011 & 0.013 & 0.009 & 0.015 & 0.012 & 0.008 & 0.013 & 0.023 & 0.021 \\
Std Err & 0.006 & 0.005 & 0.003 & 0.004 & 0.003 & 0.004 & 0.004 & 0.002 & 0.004 & 0.007 & 0.006
\end{tabular}


Table S2. Experimental Bond Angles (in degrees) of ATMO-containing Structures in the Cambridge Structural Database

\begin{tabular}{llllllll} 
CSD code & N2-C1-N1 & C1-N1-C2 & N1-C2-C3 & C2-C3-S1 & C3-S1-C1 & S1-C1-N1 & C2-C4-C5 \\
\hline BAGSAD & 124.60 & 114.92 & 112.29 & 111.58 & 90.99 & 110.21 & 120.99 \\
BAGSEH1 & 126.14 & 114.72 & 112.31 & 111.54 & 90.30 & 111.12 & 118.19 \\
BAGSEH2 & 125.10 & 112.58 & 113.03 & 112.23 & 90.00 & 112.15 & 116.66 \\
CFTXIM & 125.29 & 110.39 & 115.88 & 110.37 & 89.07 & 114.28 & 118.30 \\
EAMTHZ & 124.17 & 114.03 & 111.87 & 112.08 & 90.91 & 111.51 & 117.65 \\
EATZAC & 124.03 & 109.96 & 115.53 & 110.75 & 88.90 & 114.85 & 118.22 \\
FAJMAE & 122.25 & 109.10 & 116.61 & 110.16 & 89.45 & 114.64 & 118.71 \\
FAJMEI & 123.94 & 113.46 & 113.32 & 110.97 & 90.89 & 111.34 & 120.41 \\
FUPKOQ & 125.20 & 109.96 & 115.45 & 111.57 & 87.91 & 115.10 & 118.21 \\
GAFTIQ & 124.51 & 110.95 & 114.31 & 111.72 & 87.48 & 115.52 & 123.28 \\
GOHRAW & 124.99 & 112.80 & 112.39 & 112.08 & 89.96 & 112.76 & 116.88 \\
VENWIU & 126.51 & 115.00 & 112.39 & 112.15 & 90.46 & 109.96 & 115.72 \\
Mean & 124.73 & 112.32 & 113.78 & 111.43 & 89.69 & 112.79 & 118.60 \\
Std Dev & 1.11 & 2.16 & 1.68 & 0.71 & 1.16 & 2.01 & 2.08 \\
Std Err & 0.32 & 0.62 & 0.49 & 0.21 & 0.34 & 0.58 & 0.60 \\
& & & & & & & \\
CSD code & $\mathrm{N} 1-\mathrm{C} 2-\mathrm{C} 4$ & $\mathrm{C} 4-\mathrm{C} 5-\mathrm{O} 2$ & $\mathrm{C} 2-\mathrm{C} 4-\mathrm{N} 3$ & $\mathrm{C} 5-\mathrm{C} 4-\mathrm{N} 3$ & $\mathrm{C} 4-\mathrm{N} 3-\mathrm{O} 1$ & $\mathrm{~N} 2-\mathrm{C} 1-\mathrm{S} 1$ & $\mathrm{C} 3-\mathrm{C} 2-\mathrm{C} 4$ \\
\hline & & & & & & & \\
BAGSAD & 118.91 & 120.12 & 127.05 & 111.96 & 112.83 & 125.19 & 128.76 \\
BAGSEH1 & 120.55 & 115.84 & 116.81 & 124.99 & 110.19 & 122.70 & 127.14 \\
BAGSEH2 & 120.99 & 115.84 & 117.36 & 125.90 & 110.43 & 122.74 & 125.96 \\
CFTXIM & 120.01 & 123.38 & 119.37 & 122.33 & 110.12 & 120.43 & 124.07 \\
EAMTHZ & 120.80 & 123.37 & 129.13 & 113.17 & 112.90 & 124.31 & 127.33 \\
EATZAC & 116.65 & 124.60 & 131.01 & 110.76 & 111.66 & 121.09 & 127.80 \\
FAJMAE & 119.02 & 121.11 & 114.78 & 126.51 & 109.90 & 123.09 & 124.37 \\
FAJMEI & 118.37 & 119.96 & 116.86 & 122.67 & 111.11 & 124.72 & 128.31 \\
FUPKOQ & 119.29 & 125.30 & 120.66 & 121.05 & 110.12 & 119.69 & 124.95 \\
GAFTIQ & 118.45 & 119.59 & 116.52 & 120.06 & 110.03 & 119.90 & 127.21
\end{tabular}




$\begin{array}{llllllll}\text { GOHRAW } & 118.51 & 120.05 & 117.92 & 125.00 & 110.55 & 122.25 & 129.09 \\ \text { VENWIU } & 117.82 & 116.45 & 116.55 & 127.69 & 108.90 & 123.46 & 129.62 \\ \text { Mean } & 119.11 & 120.47 & 120.33 & 121.01 & 110.73 & 122.46 & 127.05 \\ \text { Std Dev } & 1.29 & 3.28 & 5.53 & 5.91 & 1.20 & 1.85 & 1.85 \\ \text { Std Err } & 0.37 & 0.95 & 1.60 & 1.71 & 0.35 & 0.54 & 0.53\end{array}$


Table S3. Experimental Dihedral Angles (in degrees) of ATMO-containing Structures in the Cambridge Structural Database

\begin{tabular}{|c|c|c|c|c|c|c|}
\hline CSD code & $\mathrm{N} 2-\mathrm{C} 1-\mathrm{N} 1-\mathrm{C} 2$ & $\mathrm{C} 1-\mathrm{N} 1-\mathrm{C} 2-\mathrm{C} 3$ & N1-C2-C3-S1 & C2-C3-S1-C1 & C3-S1-C1-N1 & S1-C1-N1-C2 \\
\hline BAGSAD & -179.60 & 0.53 & -1.08 & 1.06 & 0.75 & 0.29 \\
\hline BAGSEH1 & -177.80 & 0.50 & -0.77 & 0.66 & -0.38 & 0.01 \\
\hline BAGSEH2 & 178.61 & 0.61 & -0.62 & 0.37 & -0.02 & -0.32 \\
\hline CFTXIM & 179.07 & 1.08 & -1.26 & 0.82 & -0.24 & -0.40 \\
\hline EAMTHZ & -179.96 & -1.17 & 0.82 & -0.24 & -0.42 & 0.98 \\
\hline EATZAC & -178.08 & 0.32 & -0.75 & 0.72 & -0.58 & 0.27 \\
\hline FAJMAE & -176.55 & -1.75 & 0.69 & 0.40 & -1.46 & 2.02 \\
\hline FAJMEI & 178.73 & 1.87 & -0.86 & -0.19 & 1.25 & -1.97 \\
\hline FUPKOQ & 179.64 & -0.74 & -0.02 & 0.55 & -1.02 & 1.17 \\
\hline GAFTIQ & 178.43 & -1.61 & 1.08 & -0.23 & -0.71 & 1.43 \\
\hline GOHRAW & 178.11 & 1.03 & -0.05 & -0.66 & 1.27 & -1.53 \\
\hline VENWIU & -176.99 & -1.32 & 1.50 & -1.05 & 0.29 & 0.49 \\
\hline CSD code & $\mathrm{C} 1-\mathrm{N} 1-\mathrm{C} 2-\mathrm{C} 4$ & $\mathrm{~N} 1-\mathrm{C} 2-\mathrm{C} 4-\mathrm{C} 5$ & $\mathrm{~N} 1-\mathrm{C} 2-\mathrm{C} 4-\mathrm{N} 3$ & $\mathrm{C} 2-\mathrm{C} 4-\mathrm{N} 3-\mathrm{O} 1$ & $\mathrm{C} 2-\mathrm{C} 4-\mathrm{C} 5-\mathrm{O} 2$ & $\mathrm{C} 5-\mathrm{C} 4-\mathrm{N} 3-\mathrm{O} 1$ \\
\hline BAGSAD & -177.22 & 1.52 & -177.38 & -3.54 & 174.42 & 177.47 \\
\hline BAGSEH1 & -179.35 & -175.62 & 4.86 & -179.64 & 79.69 & 0.88 \\
\hline BAGSEH2 & -178.04 & 172.37 & -4.53 & 178.88 & -86.41 & 2.29 \\
\hline CFTXIM & -176.78 & -163.64 & 16.63 & 177.34 & 101.71 & -2.38 \\
\hline EAMTHZ & 179.39 & 178.11 & 0.84 & -1.87 & -4.84 & -179.24 \\
\hline EATZAC & -178.13 & 12.43 & -167.57 & -1.03 & -103.30 & 178.97 \\
\hline FAJMAE & 178.63 & -162.87 & 16.95 & 179.49 & -60.36 & -0.70 \\
\hline FAJMEI & -177.88 & 169.18 & -8.01 & 177.69 & 85.31 & 0.57 \\
\hline FUPKOQ & -174.66 & 35.44 & -147.81 & -172.61 & -89.00 & 4.06 \\
\hline GAFTIQ & 176.48 & 171.54 & -4.18 & 178.27 & -84.11 & 2.40 \\
\hline GOHRAW & -179.64 & 175.60 & 0.40 & 177.94 & -92.54 & 3.16 \\
\hline VENWIU & -176.97 & 175.71 & -2.26 & -179.52 & -66.03 & 2.79 \\
\hline
\end{tabular}




\begin{tabular}{lll} 
CSD code & N2-C1-S1-C3 & O2-C5-C \\
\hline & & \\
BAGSAD & 179.14 & -6.53 \\
BAGSEH1 & 177.52 & -100.84 \\
BAGSEH2 & -178.98 & 90.19 \\
CFTXIM & -179.73 & -78.56 \\
EAMTHZ & -179.48 & 172.85 \\
EATZAC & 177.82 & 76.70 \\
FAJMAE & 177.10 & 119.84 \\
FAJMEI & -179.46 & -97.67 \\
FUPKOQ & -179.58 & 94.25 \\
GAFTIQ & -177.86 & 91.46 \\
GOHRAW & -178.38 & 82.28 \\
VENWIU & 177.86 & 111.67
\end{tabular}


Table S4. Optimized Bond Lengths (in angstroms) from Computational Chemistry Methods

\begin{tabular}{|c|c|c|c|c|c|c|c|c|c|c|c|}
\hline Method & $\mathrm{N} 2-\mathrm{C} 1$ & $\mathrm{C} 1-\mathrm{N} 1$ & $\mathrm{~N} 1-\mathrm{C} 2$ & $\mathrm{C} 2-\mathrm{C} 3$ & C3-S1 & $\mathrm{S} 1-\mathrm{C} 1$ & $\mathrm{C} 2-\mathrm{C} 4$ & $\mathrm{C} 4-\mathrm{N} 3$ & $\mathrm{~N} 3-\mathrm{O} 1$ & $\mathrm{C} 4-\mathrm{C} 5$ & $\mathrm{C} 5-\mathrm{O} 2$ \\
\hline AM1 & 1.398 & 1.347 & 1.394 & 1.391 & 1.664 & 1.750 & 1.467 & 1.306 & 1.318 & 1.521 & 1.242 \\
\hline PM3 & 1.410 & 1.341 & 1.411 & 1.369 & 1.730 & 1.776 & 1.468 & 1.295 & 1.407 & 1.512 & 1.220 \\
\hline MNDO & 1.425 & 1.346 & 1.394 & 1.401 & 1.719 & 1.736 & 1.478 & 1.311 & 1.299 & 1.517 & 1.228 \\
\hline MMFF94 (Spartan) & 1.336 & 1.309 & 1.395 & 1.384 & 1.712 & 1.714 & 1.460 & 1.308 & 1.402 & 1.529 & 1.222 \\
\hline Sybyl (Spartan) & 1.299 & 1.264 & 1.441 & 1.341 & 1.799 & 1.773 & 1.482 & 1.277 & 1.406 & 1.480 & 1.221 \\
\hline Tripos (SYBYL) & 1.331 & 1.265 & 1.440 & 1.340 & 1.799 & 1.773 & 1.481 & 1.276 & 1.406 & 1.474 & 1.221 \\
\hline MMFF94 (SYBYL) & 1.342 & 1.310 & 1.400 & 1.383 & 1.711 & 1.714 & 1.464 & 1.305 & 1.402 & 1.533 & 1.223 \\
\hline MMFF94s (SYBYL) & 1.336 & 1.310 & 1.400 & 1.384 & 1.711 & 1.714 & 1.465 & 1.306 & 1.402 & 1.533 & 1.223 \\
\hline AMBER* & 1.421 & 1.282 & 1.391 & 1.320 & 1.704 & 1.728 & 1.490 & 1.279 & 1.410 & 1.509 & 1.230 \\
\hline OPLS/A & 1.412 & 1.281 & 1.420 & 1.393 & 1.709 & 1.725 & 1.489 & 1.278 & 1.408 & 1.490 & 1.229 \\
\hline OPLS-AA & 1.372 & 1.329 & 1.416 & 1.376 & 1.756 & 1.745 & 1.522 & 1.487 & 1.405 & 1.434 & 1.235 \\
\hline MMFF94 (MMod) & 1.343 & 1.309 & 1.399 & 1.384 & 1.712 & 1.713 & 1.464 & 1.305 & 1.401 & 1.530 & 1.223 \\
\hline MMFF94s (MMod) & 1.336 & 1.310 & 1.400 & 1.384 & 1.711 & 1.714 & 1.465 & 1.306 & 1.402 & 1.533 & 1.223 \\
\hline DFT $6-31 G^{*}$ & 1.376 & 1.299 & 1.396 & 1.372 & 1.742 & 1.764 & 1.479 & 1.294 & 1.381 & 1.539 & 1.224 \\
\hline DFT 6-31G*/SCRF & 1.365 & 1.307 & 1.394 & 1.368 & 1.742 & 1.771 & 1.478 & 1.296 & 1.380 & 1.532 & 1.239 \\
\hline DFT $6-31 G^{* *}$ & 1.374 & 1.300 & 1.396 & 1.372 & 1.742 & 1.764 & 1.479 & 1.294 & 1.381 & 1.540 & 1.224 \\
\hline DFT 6-31+G* & 1.378 & 1.300 & & & & 1.761 & & & & & 1.227 \\
\hline DFT 6-311G* & 1.374 & 1.296 & 1.395 & 1.368 & 1.740 & 1.763 & 1.478 & 1.289 & 1.378 & 1.540 & 1.217 \\
\hline DFT $6-311+\mathrm{G}^{* *}$ & 1.374 & 1.296 & 1.395 & 1.368 & 1.740 & 1.761 & 1.479 & 1.288 & 1.379 & 1.540 & 1.219 \\
\hline HF STO-3G & 1.433 & 1.303 & & & 1.734 & & 1.503 & & & & 1.220 \\
\hline HF $3-21 G(*)$ & 1.349 & 1.287 & 1.405 & 1.345 & 1.732 & 1.743 & 1.476 & 1.266 & 1.419 & 1.539 & 1.219 \\
\hline HF3-21G(*)/SCRF & 1.337 & 1.297 & 1.406 & 1.345 & 1.729 & 1.747 & 1.470 & 1.268 & 1.419 & 1.536 & 1.234 \\
\hline HF $6-31 G^{*}$ & 1.372 & 1.273 & 1.383 & 1.338 & 1.736 & 1.747 & 1.481 & 1.258 & 1.354 & 1.517 & 1.198 \\
\hline HF 6-31G** & 1.369 & 1.274 & 1.383 & 1.338 & 1.736 & 1.748 & 1.481 & 1.258 & 1.355 & 1.517 & 1.198 \\
\hline HF 6-31+G* & 1.372 & 1.274 & 1.383 & 1.339 & 1.736 & 1.745 & 1.481 & 1.258 & 1.354 & 1.518 & 1.200 \\
\hline HF 6-311G* & 1.373 & 1.270 & 1.382 & 1.336 & 1.736 & 1.746 & 1.481 & 1.256 & 1.349 & 1.519 & 1.192 \\
\hline LMP2 6-31G* & 1.388 & 1.308 & 1.400 & 1.377 & 1.731 & 1.748 & 1.482 & 1.304 & 1.402 & 1.532 & 1.234 \\
\hline
\end{tabular}


Table S5. Optimized Bond Angles (in degrees) from Computational Chemistry Methods

\begin{tabular}{|c|c|c|c|c|c|c|c|}
\hline Method & $\mathrm{N} 2-\mathrm{C} 1-\mathrm{N} 1$ & C1-N1-C2 & $\mathrm{N} 1-\mathrm{C} 2-\mathrm{C} 3$ & $\mathrm{C} 2-\mathrm{C} 3-\mathrm{S} 1$ & $\mathrm{C} 3-\mathrm{S} 1-\mathrm{C} 1$ & $\mathrm{~S} 1-\mathrm{C} 1-\mathrm{N} 1$ & $\mathrm{C} 2-\mathrm{C} 4-\mathrm{C} 5$ \\
\hline AM1 & 126.631 & 109.433 & 114.994 & 111.325 & 90.688 & 113.561 & 114.535 \\
\hline PM3 & 120.382 & 110.809 & 114.420 & 112.070 & 88.854 & 113.847 & 116.197 \\
\hline MNDO & 124.624 & 110.121 & 115.051 & 109.892 & 90.401 & 114.478 & 117.688 \\
\hline MMFF94 (Spartan) & 123.134 & 110.694 & 113.790 & 110.408 & 89.713 & 115.395 & 118.548 \\
\hline Sybyl (Spartan) & 123.936 & 117.516 & 108.459 & 114.134 & 86.790 & 113.045 & 119.064 \\
\hline Tripos (SYBYL) & 124.510 & 117.631 & 108.516 & 114.076 & 86.904 & 112.873 & 117.573 \\
\hline MMFF94 (SYBYL) & 123.134 & 110.465 & 113.790 & 110.408 & 89.771 & 115.510 & 118.605 \\
\hline MMFF94s (SYBYL) & 123.191 & 110.522 & 113.790 & 110.465 & 89.771 & 115.452 & 118.834 \\
\hline AMBER* & 120.898 & 109.318 & 113.561 & 113.790 & 86.102 & 117.172 & 120.038 \\
\hline OPLS/A & 119.866 & 109.204 & 112.127 & 112.643 & 87.191 & 118.892 & 120.497 \\
\hline OPLS-AA & 123.076 & 110.236 & 112.070 & 113.962 & 86.217 & 117.516 & 126.516 \\
\hline MMFF94 (MMod) & 123.076 & 110.580 & 113.675 & 110.465 & 89.713 & 115.510 & 118.204 \\
\hline MMFF94s (MMod) & 123.191 & 110.522 & 113.790 & 110.465 & 89.771 & 115.452 & 118.834 \\
\hline DFT $6-31 G^{*}$ & 124.166 & 112.013 & 114.134 & 110.866 & 88.510 & 114.478 & 123.707 \\
\hline DFT 6-31G*/SCRF & 124.510 & 111.726 & 115.051 & 110.408 & 88.854 & 113.962 & 123.535 \\
\hline DFT 6-31G** & 124.166 & 112.070 & 114.191 & 110.809 & 88.510 & 114.420 & 123.822 \\
\hline DFT $6-31+\mathrm{G}^{*}$ & 124.051 & 112.013 & 114.076 & 110.809 & 88.567 & 114.478 & 123.707 \\
\hline DFT 6-311G* & 124.223 & 112.185 & 114.134 & 110.866 & 88.452 & 114.363 & 123.478 \\
\hline DFT $6-311+\mathrm{G}^{* *}$ & 124.223 & 112.127 & 114.248 & 110.752 & 88.567 & 114.363 & 123.363 \\
\hline HF STO-3G & 122.446 & 108.745 & 114.707 & 111.841 & 87.994 & 116.713 & 121.127 \\
\hline HF $3-21 G(*)$ & 124.108 & 112.529 & 113.503 & 111.153 & 88.968 & 113.847 & 123.191 \\
\hline HF $3-21 G\left({ }^{*}\right) / \mathrm{SCRF}$ & 124.854 & 112.242 & 114.076 & 110.866 & 89.427 & 113.389 & 122.962 \\
\hline HF 6-31G* & 123.994 & 110.866 & 116.255 & 109.548 & 88.338 & 114.994 & 117.229 \\
\hline HF $6-31 G^{* *}$ & 123.994 & 110.866 & 116.312 & 109.490 & 88.395 & 114.994 & 117.229 \\
\hline HF $6-31+\mathrm{G}^{*}$ & 123.936 & 110.981 & 116.140 & 109.490 & 88.452 & 114.936 & 117.057 \\
\hline HF 6-311G* & 123.994 & 110.981 & 116.140 & 109.490 & 88.338 & 114.994 & 117.229 \\
\hline LMP2 6-31G* & 123.478 & 110.981 & 114.306 & 110.694 & 88.854 & 115.051 & 123.248 \\
\hline
\end{tabular}




\begin{tabular}{|c|c|c|c|c|c|c|c|}
\hline Method & $\mathrm{N} 1-\mathrm{C} 2-\mathrm{C} 4$ & $\mathrm{C} 4-\mathrm{C} 5-\mathrm{O} 2$ & $\mathrm{C} 2-\mathrm{C} 4-\mathrm{N} 3$ & $\mathrm{C} 5-\mathrm{C} 4-\mathrm{N} 3$ & C4-N3-O1 & $\mathrm{N} 2-\mathrm{C} 1-\mathrm{S} 1$ & $\mathrm{C} 3-\mathrm{C} 2-\mathrm{C} 4$ \\
\hline AM1 & 120.669 & 120.841 & 128.809 & 116.599 & 117.520 & 119.580 & 124.338 \\
\hline PM3 & 119.465 & 122.790 & 126.917 & 116.885 & 118.950 & 125.484 & 126.115 \\
\hline MNDO & 117.631 & 122.675 & 128.121 & 114.191 & 118.150 & 120.898 & 127.318 \\
\hline MMFF94 (Spartan) & 120.153 & 120.439 & 125.943 & 115.510 & 113.450 & 121.471 & 126.057 \\
\hline Sybyl (Spartan) & 120.554 & 117.287 & 125.541 & 115.395 & 110.010 & 122.962 & 130.987 \\
\hline Tripos (SYBYL) & 120.955 & 120.038 & 126.172 & 116.255 & 109.720 & 122.618 & 130.586 \\
\hline MMFF94 (SYBYL) & 120.153 & 120.038 & 125.369 & 116.025 & 113.730 & 121.357 & 126.000 \\
\hline MMFF94s (SYBYL) & 120.325 & 120.096 & 125.255 & 115.911 & 113.730 & 121.357 & 125.885 \\
\hline AMBER* & 122.904 & 119.924 & 121.471 & 118.490 & 125.830 & 121.930 & 123.478 \\
\hline OPLS/A & 124.280 & 120.325 & 121.070 & 118.376 & 125.250 & 121.242 & 123.535 \\
\hline OPLS-AA & 119.408 & 121.815 & 112.127 & 121.127 & 108.570 & 119.408 & 128.522 \\
\hline MMFF94 (MMod) & 119.924 & 119.981 & 126.000 & 115.796 & 113.850 & 121.414 & 126.401 \\
\hline MMFF94s (MMod) & 120.325 & 120.153 & 125.255 & 115.911 & 113.730 & 121.357 & 125.885 \\
\hline DFT $6-31 G^{*}$ & 119.350 & 120.726 & 126.631 & 109.662 & 114.710 & 121.242 & 126.459 \\
\hline DFT 6-31G*/SCRF & 119.121 & 120.210 & 125.771 & 110.694 & 114.540 & 121.471 & 125.828 \\
\hline DFT $6-31 \mathrm{G}^{* *}$ & 119.350 & 120.669 & 126.516 & 109.662 & 114.710 & 121.299 & 126.459 \\
\hline DFT 6-31+G* & 119.465 & 120.783 & 126.344 & 109.949 & 114.940 & 121.299 & 126.401 \\
\hline DFT 6-311G* & 119.580 & 120.726 & 126.631 & 109.892 & 114.990 & 121.299 & 126.287 \\
\hline DFT $6-311+\mathrm{G}^{* *}$ & 119.580 & 120.841 & 126.401 & 110.236 & 115.050 & 121.299 & 126.172 \\
\hline HF STO-3G & 117.917 & 121.758 & 126.573 & 112.299 & 113.160 & 120.669 & 127.376 \\
\hline HF $3-21 G(*)$ & 120.268 & 119.924 & 126.459 & 110.350 & 115.800 & 121.987 & 126.229 \\
\hline HF $3-21 G(*) / S C R F$ & 120.153 & 119.465 & 126.745 & 110.293 & 115.680 & 121.758 & 125.771 \\
\hline HF 6-31G* & 117.000 & 120.325 & 127.834 & 114.936 & 115.220 & 120.955 & 126.745 \\
\hline HF 6-31G** & 116.943 & 120.325 & 127.834 & 114.879 & 115.170 & 120.955 & 126.745 \\
\hline HF $6-31+\mathrm{G}^{*}$ & 116.656 & 120.153 & 128.006 & 114.936 & 115.220 & 121.070 & 127.204 \\
\hline HF 6-311G* & 117.000 & 120.268 & 127.777 & 114.936 & 115.450 & 120.955 & 126.860 \\
\hline LMP2 6-31G* & 118.949 & 120.611 & 126.745 & 109.949 & 113.220 & 121.299 & 126.745 \\
\hline
\end{tabular}


TABLE S6. Single-Point Energies (in eV) for Variation of the C6-O1-N3-C4 Dihedral Angle in 30 Increments

\begin{tabular}{|c|c|c|c|c|c|c|c|c|c|c|}
\hline Method & $0^{\circ}$ & $30^{\circ}$ & $60^{\circ}$ & $90^{\circ}$ & $120^{\circ}$ & $150^{\circ}$ & $180^{\circ}$ & $210^{\circ}$ & $240^{\circ}$ & $270^{\circ}$ \\
\hline AM1 & ND & 17.446 & 8.3141 & 1.3212 & 0.926 & 0.8182 & 0.7648 & 0.8083 & 0.9169 & 1.018 \\
\hline PM3 & 2.95172 & 2.005 & 1.2125 & 0.9711 & 0.8736 & 0.7802 & 0.7424 & 0.781 & 0.8695 & 0.9994 \\
\hline MNDO & 2.82215 & 2.2145 & 0.9453 & 0.2594 & 0.0325 & -0.1077 & -0.1623 & -0.1081 & 0.0262 & 0.248 \\
\hline MMFF94 (Spartan) & 118.015 & 73.105 & 140.94 & 4.0607 & 1.6124 & 1.4531 & 1.4057 & 1.4541 & 1.5583 & 1.7069 \\
\hline Sybyl (Spartan) & 191819 & 86366 & 329496 & 27.754 & 0.5125 & 0.5095 & 0.5264 & 0.514 & 0.483 & 0.4745 \\
\hline AMBER* & 330.259 & 155.46 & 101.05 & 6.6581 & 4.337 & 4.1136 & 4.0056 & 4.0988 & 4.2958 & 4.4055 \\
\hline OPLS/A & 613.995 & 6140.8 & 163.27 & 5.552 & 1.3673 & 1.2298 & 1.1699 & 1.2254 & 1.3434 & 1.4436 \\
\hline OPLS-AA & 16.5336 & 4.1348 & 2.6357 & 2.2045 & 2.103 & 2.0826 & 2.0978 & 2.1884 & 111.62 & 92.819 \\
\hline MMFF94 (MMod) & 23.5839 & 47.097 & 16.435 & 5.4088 & 4.5174 & 4.3747 & 4.3226 & 4.3699 & 4.4849 & 4.6892 \\
\hline MMFF94s (MMod) & 23.3579 & 41.76 & 17.634 & 6.0331 & 4.8753 & 4.7256 & 4.6736 & 4.7209 & 4.8345 & 5.0241 \\
\hline Tripos & 191779 & 86301.4 & 345006 & 28.248 & 0.524 & 0.5204 & 0.5373 & 0.5249 & 0.4939 & 0.4852 \\
\hline MMFF94 (SYBYL) & 121.115 & 73.371 & 144.22 & 4.4131 & 1.6744 & 1.4992 & 1.4455 & 1.4932 & 1.6048 & 1.7715 \\
\hline MMFF94s (SYBYL) & 122.819 & 74.344 & 146.49 & 4.4799 & 1.6687 & 1.4926 & 1.4386 & 1.4856 & 1.5954 & 1.7594 \\
\hline Method & $300^{\circ}$ & $330^{\circ}$ & & & & & & & & \\
\hline AM1 & 1.3552 & 7.1375 & & & & & & & & \\
\hline PM3 & 1.5603 & 2.6167 & & & & & & & & \\
\hline MNDO & 0.9843 & 2.1892 & & & & & & & & \\
\hline MMFF94 (Spartan) & 2.8441 & 20.349 & & & & & & & & \\
\hline Sybyl (Spartan) & 3.2695 & 3372 & & & & & & & & \\
\hline AMBER* & 4.5118 & 17.488 & & & & & & & & \\
\hline OPLS/A & 2.162 & 27.245 & & & & & & & & \\
\hline OPLS-AA & 55.067 & 140.71 & & & & & & & & \\
\hline MMFF94 (MMod) & 5.9342 & 14.913 & & & & & & & & \\
\hline MMFF94s (MMod) & 6.1129 & 13.642 & & & & & & & & \\
\hline Tripos & 3.2392 & 3350 & & & & & & & & \\
\hline MMFF94 (SYBYL) & 2.9243 & 19.932 & & & & & & & & \\
\hline MMFF94s (SYBYL) & 2.9168 & 20.054 & & & & & & & & \\
\hline
\end{tabular}




\begin{tabular}{|c|c|c|c|c|c|c|}
\hline Method & $0^{\circ}$ & $30^{\circ}$ & $60^{\circ}$ & $90^{\circ}$ & $120^{\circ}$ & $150^{\circ}$ \\
\hline HF STO-3G & -27828.4012 & -27823.2861 & -27847.3877 & -27856.9494 & -27857.5962 & -27857.7831 \\
\hline HF $3-21 G(*)$ & -28011.6674 & -28014.2663 & -28045.7694 & -28048.6102 & -28048.9118 & -28049.0832 \\
\hline HF $3-21 G(*) / S C R F$ & -28012.1636 & -28013.0287 & -28046.6635 & -28049.7436 & -28049.9663 & -28050.0659 \\
\hline HF 6-31G* & -28179.9008 & -28189.739 & -28195.3541 & -28196.0786 & -28196.2157 & -28196.3357 \\
\hline HF 6-31G** & -28180.7015 & -28190.3679 & -28195.9883 & -28196.7095 & -28196.8461 & -28196.9661 \\
\hline HF $6-31+\mathrm{G}^{*}$ & -28179.9383 & -28189.8323 & -28195.7796 & -28196.5603 & -28196.7124 & -28196.8386 \\
\hline HF 6-311G* & -28183.9946 & -28193.9741 & -28199.7123 & -28200.4531 & -28200.5911 & -28200.714 \\
\hline DFT 6-31G* & -28295.8203 & -28300.5323 & -28316.7051 & -28318.009 & -28318.2377 & -28318.4368 \\
\hline DFT $6-31 G * / S C R F$ & ND & -28251.6092 & -28310.7402 & -28314.1868 & -28314.5209 & -28314.65 \\
\hline DFT $6-31 \mathrm{G}^{* *}$ & -28296.5497 & -28301.0088 & -28317.2703 & -28318.5541 & -28318.7828 & -28318.9821 \\
\hline DFT 6-311G* & -28301.4916 & -28307.3059 & -28321.7598 & -28322.958 & -28323.1868 & -28323.3884 \\
\hline DFT 6-31+G* & -28297.5881 & -28303.5886 & -28317.6012 & -28318.7826 & -28319.0209 & -28319.2291 \\
\hline DFT $6-311+\mathrm{G}^{* *}$ & -28303.3171 & -28310.7243 & -28322.8972 & -28323.9471 & -28324.1823 & -28324.3869 \\
\hline LMP2 6-31G* & ND & -28179.8915 & -28241.2151 & -28244.9035 & -28245.2221 & -28245.3799 \\
\hline Method & $180^{\circ}$ & $210^{\circ}$ & $240^{\circ}$ & $270^{\circ}$ & $300^{\circ}$ & $330^{\circ}$ \\
\hline HF STO-3G & -27857.8689 & -27857.8044 & -27857.6421 & -27857.4687 & -27856.3942 & -27844.5295 \\
\hline HF $3-21 G(*)$ & -28049.1535 & -28049.0845 & -28048.9144 & -28048.6159 & -28045.8208 & -28012.175 \\
\hline HF $3-21 G\left({ }^{*}\right) / S C R F$ & -28050.1132 & -28050.0652 & -28049.9741 & -28049.7851 & -28047.3591 & -28017.4358 \\
\hline HF $6-31 G^{*}$ & -28196.3811 & -28196.3123 & -28196.1591 & -28195.5614 & -28187.7933 & -28182.0586 \\
\hline HF 6-31G** & -28197.0117 & -28196.9432 & -28196.79 & -28196.2065 & -28188.645 & -28182.7535 \\
\hline $\mathrm{HF} 6-31+\mathrm{G}^{*}$ & -28196.8868 & -28196.8158 & -28196.6614 & -28196.1272 & -28188.9884 & -28182.1034 \\
\hline HF 6-311G* & -28200.759 & -28200.6869 & -28200.5295 & -28199.9572 & -28192.4177 & -28186.1915 \\
\hline DFT 6-31G* & -28318.5131 & -28318.4075 & -28318.1792 & -28317.8206 & -28313.8811 & -28285.6481 \\
\hline DFT $6-31 G * / S C R F$ & -28314.7013 & -28314.6475 & -28314.5167 & -28314.181 & -28310.7173 & -28251.5436 \\
\hline DFT 6-31G** & -28319.0584 & -28318.9522 & -28318.7225 & -28318.3666 & -28314.5802 & -28286.1034 \\
\hline DFT 6-311G* & -28323.461 & -28323.3492 & -28323.1135 & -28322.733 & -28318.4064 & -28291.8371 \\
\hline DFT $6-31+\mathrm{G}^{*}$ & -28319.3037 & -28319.1901 & -28318.9477 & -28318.5495 & -28314.0466 & -28287.5868 \\
\hline
\end{tabular}




$\begin{array}{lllllll}\text { DFT 6-311+G** } & -28324.2536 & -28324.338 & -28324.0951 & -28323.6812 & -28318.7657 & -28294.1522 \\ \text { LMP2 6-31G* } & -28245.4483 & -28245.3737 & -28245.213 & -28244.8942 & -28241.1849 & -28179.8353\end{array}$

ND stands for no data as explained in the text. 
Table S7. Single-Point Energies (in eV) for Variation of the N1-C2-C4-N3 Dihedral Angle in $30^{\circ}$ Increments

\begin{tabular}{|c|c|c|c|c|c|c|c|c|}
\hline Method & $0^{\circ}$ & $30^{\circ}$ & $60^{\circ}$ & $90^{\circ}$ & $120^{\circ}$ & $150^{\circ}$ & $180^{\circ}$ & $210^{\circ}$ \\
\hline AM1 & 0.93693 & 0.8628 & 0.8317 & 0.8283 & 0.7982 & 0.7652 & 0.7964 & 0.8503 \\
\hline PM3 & 0.87433 & 0.8079 & 0.7462 & 0.7483 & 0.7707 & 0.8139 & 0.8778 & 0.8664 \\
\hline MNDO & 0.06962 & -0.0161 & -0.131 & -0.163 & -0.132 & -0.0343 & 0.0746 & 0.0161 \\
\hline MMFF94 (Spartan) & 1.50152 & 1.4672 & 1.4976 & 1.5415 & 1.4614 & 1.417 & 1.6089 & 1.5853 \\
\hline Sybyl (Spartan) & 63.9101 & 2.8812 & 0.603 & 0.637 & 0.5685 & 0.495 & 0.6738 & 0.5187 \\
\hline AMBER* & 7.56648 & 2.2678 & 2.0362 & 2.0921 & 2.0418 & 2.0054 & 2.2552 & 2.0323 \\
\hline OPLS/A & 1.43886 & 0.3791 & 0.2529 & 0.2728 & 0.2069 & 0.2685 & 0.9161 & 0.3253 \\
\hline OPLS-AA & 3.02911 & 3.0261 & 3.2668 & 3.2894 & 2.8539 & 2.3025 & 2.0843 & 2.3984 \\
\hline MMFF94 (MMod) & 1.84054 & 1.7408 & 1.7321 & 1.6991 & 1.5386 & 1.4432 & 1.5894 & 1.6015 \\
\hline MMFF94s (MMod) & 1.90084 & 1.7343 & 1.7069 & 1.667 & 1.5104 & 1.451 & 1.6466 & 1.6154 \\
\hline Tripos & 64.2686 & 2.9669 & 0.6141 & 0.6479 & 0.5797 & 0.506 & 0.684 & 0.5304 \\
\hline MMFF94 (SYBYL) & 1.97276 & 1.7719 & 1.7345 & 1.688 & 1.5221 & 1.4575 & 1.6592 & 1.6478 \\
\hline MMFF94s (SYBYL) & 1.90179 & 1.7346 & 1.7071 & 1.6668 & 1.5101 & 1.4512 & 1.4737 & 1.6158 \\
\hline Method & $240^{\circ}$ & $270^{\circ}$ & $300^{\circ}$ & $330^{\circ}$ & & & & \\
\hline AM1 & 0.921 & 0.934 & 0.9221 & 0.9414 & & & & \\
\hline PM3 & 0.8375 & 0.8085 & 0.7981 & 0.8397 & & & & \\
\hline MNDO & -0.058 & -0.088 & -0.073 & 0.0079 & & & & \\
\hline MMFF94 (Spartan) & 1.6327 & 1.6941 & 1.6165 & 1.5172 & & & & \\
\hline Sybyl (Spartan) & 0.575 & 0.6373 & 0.5861 & 1.447 & & & & \\
\hline AMBER* & 2.0392 & 2.0943 & 2.0392 & 2.1693 & & & & \\
\hline OPLS/A & 0.2629 & 0.3232 & 0.321 & 0.6967 & & & & \\
\hline OPLS-AA & 3.0187 & 3.4091 & 3.361 & 3.1462 & & & & \\
\hline MMFF94 (MMod) & 1.736 & 1.9043 & 2.0136 & 2.0115 & & & & \\
\hline MMFF94s (MMod) & 1.7204 & 1.8883 & 2.0648 & 2.2383 & & & & \\
\hline Tripos & 0.5864 & 0.6482 & 0.5965 & 1.4389 & & & & \\
\hline MMFF94 (SYBYL) & 1.7613 & 1.9318 & 2.1257 & 2.4143 & & & & \\
\hline MMFF94s (SYBYL) & 1.7201 & 1.8883 & 2.0649 & 2.2403 & & & & \\
\hline
\end{tabular}




\begin{tabular}{|c|c|c|c|c|c|c|}
\hline Method & $0^{\circ}$ & $30^{\circ}$ & $60^{\circ}$ & $90^{\circ}$ & $120^{\circ}$ & $150^{\circ}$ \\
\hline HF STO-3G & -27856.6266 & -27857.3208 & -27857.6541 & -27857.6748 & -27857.7609 & -27857.8684 \\
\hline HF $3-21 G(*)$ & -28047.6816 & -28048.3739 & -28048.6345 & -28048.641 & -28048.7654 & -28049.0223 \\
\hline HF $3-21 G(*) / S C R F$ & ND & -27951.6799 & -28035.0766 & -28040.9491 & -28043.3098 & -28044.489 \\
\hline HF $6-31 G^{*}$ & -28196.1387 & -28196.1925 & -28196.2349 & -28196.217 & -28196.1646 & -28196.0809 \\
\hline HF 6-31G** & -28196.7677 & -28196.8193 & -28196.8634 & -28196.8461 & -28196.793 & -28196.7107 \\
\hline HF $6-31+\mathrm{G}^{*}$ & -28196.6042 & -28196.6659 & -28196.7245 & -28196.7023 & -28196.6399 & -28196.5777 \\
\hline HF 6-311G* & -28200.5073 & -28200.5587 & -28200.608 & -28200.5921 & -28200.5358 & -28200.4602 \\
\hline DFT 6-31G* & -28317.8339 & -28317.8541 & -28318.148 & -28318.1711 & -28318.2418 & -28318.4046 \\
\hline DFT $6-31 G^{*} / \mathrm{SCRF}$ & ND & -28228.1222 & -28307.0277 & -28311.9923 & -28313.7025 & -28314.6084 \\
\hline DFT 6-31G** & -28318.3601 & -28318.4091 & -28318.6961 & -28318.7151 & -28318.7844 & -28318.9485 \\
\hline DFT 6-311G* & -28322.8154 & -28322.7775 & -28323.0838 & -28323.1309 & -28323.2056 & -28323.3565 \\
\hline DFT 6-31+G* & -28318.6653 & -28318.6617 & -28318.9722 & -28319.0282 & -28319.0944 & -28319.2154 \\
\hline DFT 6-311+G** & -28323.8296 & -28323.8064 & -28324.1206 & -28324.1813 & -28324.2467 & -28324.3645 \\
\hline LMP2 6-31G* & ND & -28155.6585 & -28237.2165 & -28242.4597 & -28244.4923 & -28245.4115 \\
\hline Method & $180^{\circ}$ & $210^{\circ}$ & $240^{\circ}$ & $270^{\circ}$ & $300^{\circ}$ & $330^{\circ}$ \\
\hline HF STO-3G & -27857.7324 & -27857.619 & -27857.6338 & -27857.62 & -27857.6342 & -27857.4182 \\
\hline HF 3-21G(*) & -28049.1535 & -28049.0189 & -28048.763 & -28048.6402 & -28048.6331 & -28048.3664 \\
\hline HF $3-21 G(*) / S C R F$ & -28044.8086 & -28044.5052 & -28043.3393 & -28040.9874 & -28034.1015 & -27887.4518 \\
\hline HF $6-31 G^{*}$ & -28196.1869 & -28196.3706 & -28196.3499 & -28196.2986 & -28196.3274 & -28196.2755 \\
\hline HF 6-31G** & -28196.819 & -28197.0017 & -28196.98 & -28196.9286 & -28196.9571 & -28196.9068 \\
\hline HF $6-31+\mathrm{G}^{*}$ & -28196.7102 & -28196.8798 & -28196.8537 & -28196.8017 & -28196.8233 & -28196.7626 \\
\hline HF 6-311G* & -28200.5764 & -28200.7517 & -28200.7231 & -28200.6719 & -28200.7 & -28200.6491 \\
\hline DFT 6-31G* & -28318.5055 & -28318.4709 & -28318.2854 & -28318.1601 & -28318.1856 & -28318.1634 \\
\hline DFT 6-31G*/SCRF & -28314.7016 & -28314.6275 & -28313.7428 & -28312.0087 & -28306.1715 & -28167.7551 \\
\hline DFT 6-31G** & -28319.0502 & -28319.0143 & -28318.8266 & -28318.7032 & -28318.7303 & -28318.7022 \\
\hline DFT 6-311G* & -28323.4512 & -28323.2558 & -28323.1291 & -28323.1428 & -28323.1155 & -28323.1155 \\
\hline DFT $6-31+\mathrm{G}^{*}$ & -28319.2916 & -28319.2826 & -28319.136 & -28319.0313 & -28319.0371 & -28318.9814 \\
\hline
\end{tabular}




$\begin{array}{lllllll}\text { DFT 6-311+G** } & -28324.4401 & -28324.4437 & -28324.2983 & -28324.1893 & -28324.1935 & -28324.1353 \\ \text { LMP2 6-31G* } & -28245.4483 & -28245.4234 & -28244.5179 & -28242.4956 & -28236.3646 & -28093.0216\end{array}$

ND stands for no data as explained in the text. 
Table S8. Single-Point Energies (in eV) for Variation of the O2-C4-C5-C2 Dihedral Angle in $30^{\circ}$ Increments

\begin{tabular}{|c|c|c|c|c|c|c|c|c|c|c|}
\hline Method & $0^{\circ}$ & $30^{\circ}$ & $60^{\circ}$ & $90^{\circ}$ & $120^{\circ}$ & $150^{\circ}$ & $180^{\circ}$ & $210^{\circ}$ & $240^{\circ}$ & $270^{\circ}$ \\
\hline AM1 & 1.17737 & 1.1166 & 0.9016 & 0.854 & 0.8557 & 1.0455 & 1.7218 & 1.5082 & 0.8506 & 0.777 \\
\hline PM3 & 0.88748 & 0.8903 & 0.8604 & 0.8328 & 0.8405 & 0.9065 & 0.9101 & 0.8449 & 0.7783 & 0.7451 \\
\hline MNDO & 0.07682 & 0.0466 & -0.035 & -0.065 & -0.008 & 0.1045 & 0.1601 & 0.0781 & -0.086 & -0.161 \\
\hline MMFF94 (Spartan) & 1.54037 & 1.5977 & 1.5846 & 1.576 & 1.5893 & 1.6281 & 1.6023 & 1.5096 & 1.4712 & 1.4331 \\
\hline Sybyl (Spartan) & 0.48665 & 0.543 & 0.6518 & 0.7093 & 0.6471 & 0.5351 & 0.4932 & 0.5367 & 0.6455 & 0.7091 \\
\hline AMBER* & 1.61324 & 1.6805 & 1.7924 & 1.8579 & 1.8019 & 1.6865 & 1.6375 & 1.6865 & 1.7846 & 1.8345 \\
\hline OPLS/A & 0.20995 & 0.2789 & 0.3566 & 0.3978 & 0.36 & 0.2711 & 0.1969 & 0.2013 & 0.2746 & 0.3141 \\
\hline OPLS-AA & 1.85095 & 1.8514 & 1.9551 & 2.1212 & 2.1824 & 2.1173 & 2.0861 & 2.1598 & 2.2097 & 2.1212 \\
\hline MMFF94 (MMod) & 1.58201 & 1.6024 & 1.5768 & 1.5868 & 1.6332 & 1.723 & 1.7507 & 1.5547 & 1.4419 & 1.4454 \\
\hline MMFF94s (MMod) & 1.5391 & 1.5764 & 1.5673 & 1.5729 & 1.5989 & 1.6471 & 1.6263 & 1.4814 & 1.4393 & 1.4406 \\
\hline Tripos & 0.49989 & 0.55628 & 0.6646 & 0.72155 & 0.65874 & 0.54613 & 0.50432 & 0.54817 & 0.6574 & 0.72129 \\
\hline MMFF94 (SYBYL) & 1.58366 & 1.5986 & 1.5761 & 1.5849 & 1.6238 & 1.6909 & 1.6923 & 1.5162 & 1.4467 & 1.4676 \\
\hline MMFF94s (SYBYL) & 1.5385 & 1.5763 & 1.5676 & 1.5733 & 1.5991 & 1.6472 & 1.6263 & 1.4812 & 1.4391 & 1.4403 \\
\hline Method & $300^{\circ}$ & $330^{\circ}$ & & & & & & & & \\
\hline AM1 & 0.8162 & 0.9481 & & & & & & & & \\
\hline PM3 & 0.7517 & 0.814 & & & & & & & & \\
\hline MNDO & -0.133 & -0.024 & & & & & & & & \\
\hline MMFF94 (Spartan) & 1.4059 & 1.4397 & & & & & & & & \\
\hline Sybyl (Spartan) & 0.6505 & 0.5349 & & & & & & & & \\
\hline AMBER* & 1.7664 & 1.6492 & & & & & & & & \\
\hline OPLS/A & 0.2724 & 0.2078 & & & & & & & & \\
\hline OPLS-AA & 1.959 & 1.8648 & & & & & & & & \\
\hline MMFF94 (MMod) & 1.454 & 1.5005 & & & & & & & & \\
\hline MMFF94s (MMod) & 1.4345 & 1.4649 & & & & & & & & \\
\hline Tripos & 0.66282 & 0.54756 & & & & & & & & \\
\hline MMFF94 (SYBYL) & 1.4799 & 1.5188 & & & & & & & & \\
\hline MMFF94s (SYBYL) & 1.4341 & 1.4643 & & & & & & & & \\
\hline
\end{tabular}




\begin{tabular}{|c|c|c|c|c|c|c|}
\hline Method & $0^{\circ}$ & $30^{\circ}$ & $60^{\circ}$ & $90^{\circ}$ & $120^{\circ}$ & $150^{\circ}$ \\
\hline HF STO-3G & -27857.735 & -27857.6658 & -27857.6473 & -27857.6627 & -27857.7427 & -27858.0445 \\
\hline HF $3-21 G(*)$ & -28048.9226 & -28048.9084 & -28048.8339 & -28048.7738 & -28048.8499 & -28049.0148 \\
\hline HF $3-21 G(*) / S C R F$ & ND & -27951.7909 & -28030.7669 & -28038.0127 & -28042.2192 & -28044.1478 \\
\hline HF $6-31 G^{*}$ & -28196.2529 & -28196.3779 & -28196.3323 & -28196.2172 & -28196.156 & -28196.0728 \\
\hline HF 6-31G** & -28196.881 & -28197.008 & -28196.9642 & -28196.8498 & -28196.7879 & -28196.7067 \\
\hline HF $6-31+\mathrm{G}^{*}$ & -28196.7192 & -28196.8738 & -28196.858 & -28196.7474 & -28196.6541 & -28196.5208 \\
\hline HF 6-311G* & -28200.6241 & -28200.7551 & -28200.7165 & -28200.6074 & -28200.5333 & -28200.4265 \\
\hline DFT 6-31G* & -28318.3738 & -28318.4041 & -28318.3811 & -28318.3402 & -28318.3995 & -28318.5111 \\
\hline DFT 6-31G*/SCRF & ND & -28224.8286 & -28302.9174 & -28309.3836 & -28312.5137 & -28314.0435 \\
\hline DFT $6-31 G^{* *}$ & -28318.9175 & -28318.9468 & -28318.9218 & -28318.8793 & -28318.9385 & -28319.0537 \\
\hline DFT 6-311G* & -28323.3337 & -28323.3721 & -28323.3589 & -28323.3283 & -28323.3756 & -28323.462 \\
\hline DFT 6-31+G* & -28319.1778 & -28319.2331 & -28319.2398 & -28319.2091 & -28319.2362 & -28319.3042 \\
\hline DFT 6-311+G** & -28324.3349 & -28324.393 & -28324.3977 & -28324.3639 & -28324.3892 & -28324.4594 \\
\hline LMP2 6-31G* & ND & -28152.2123 & -28233.0056 & -28239.9126 & -28243.2612 & -28244.8466 \\
\hline Method & $180^{\circ}$ & $210^{\circ}$ & $240^{\circ}$ & $270^{\circ}$ & $300^{\circ}$ & $330^{\circ}$ \\
\hline HF STO-3G & -27857.8092 & -27857.6338 & -27857.6295 & -27857.6618 & -27857.7415 & -27857.7985 \\
\hline HF $3-21 G(*)$ & -28049.1535 & -28049.016 & -28048.8503 & -28048.7746 & -28048.8358 & -28048.9106 \\
\hline HF $3-21 G(*) / S C R F$ & -28044.811 & -28044.5089 & -28042.5218 & -28035.8091 & -28025.8653 & -27952.6559 \\
\hline HF $6-31 G^{*}$ & -28196.019 & -28196.1566 & -28196.1637 & -28196.1037 & -28196.089 & -28196.1124 \\
\hline HF 6-31G** & -28196.6482 & -28196.7881 & -28196.7974 & -28196.7371 & -28196.7208 & -28196.7413 \\
\hline HF $6-31+\mathrm{G}^{*}$ & -28196.4655 & -28196.653 & -28196.6908 & -28196.6341 & -28196.5894 & -28196.5778 \\
\hline HF 6-311G* & -28200.3671 & -28200.5342 & -28200.5511 & -28200.4944 & -28200.4661 & -28200.4801 \\
\hline DFT 6-31G* & -28318.4243 & -28318.3429 & -28318.3142 & -28318.3013 & -28318.3538 & -28318.3837 \\
\hline DFT $6-31 G^{*} / \mathrm{SCRF}$ & -28314.7013 & -28314.5911 & -28312.9678 & -28307.1073 & -28298.434 & -28226.0983 \\
\hline DFT $6-31 G^{* *}$ & -28318.9772 & -28318.8908 & -28318.8575 & -28318.8425 & -28318.8945 & -28318.9256 \\
\hline DFT 6-311G* & -28323.3558 & -28323.2995 & -28323.288 & -28323.2819 & -28323.3227 & -28323.3395 \\
\hline DFT $6-31+\mathrm{G}^{*}$ & -28319.2107 & -28319.1622 & -28319.1614 & -28319.1561 & -28319.1783 & -28319.1756 \\
\hline
\end{tabular}




$\begin{array}{llllllr}\text { DFT 6-311+G** } & -28324.3491 & -28324.308 & -28324.316 & -28324.31 & -28324.3283 & -28324.3262 \\ \text { LMP2 6-31G* } & -28245.4483 & -28245.323 & -28243.702 & -28237.8767 & -28228.1617 & -28153.5788\end{array}$

ND stands for no data as explained in the text. 